decreased by $1687 \mathrm{ng} / \mathrm{mL}$ (IQR: 291 to $3992 \mathrm{ng} / \mathrm{mL}$; $\mathrm{p}=0.0229)$.

There was no significant association between change in plasma HHQ and change in FEV1 during treatment of a pulmonary exacerbation (Spearman's correlation co-efficient, $r=-0.42$; $\mathrm{p}=0.15)$.

Conclusions Plasma HHQ declined significantly during treatment of a pulmonary exacerbation and merits further investigation as a biomarker for measuring treatment response in CF. There was no significant decline in plasma NHQ during systemic antimicrobial therapy.

\section{P93 IN- VITRO ACTIVITY OF SEVEN HOSPITAL BIOCIDES AGAINST MYCOBACTERIUM ABSCESSUS}

'S Caskey, ${ }^{2} \mathrm{JE}$ Moore, ${ }^{1} \mathrm{JC}$ Rendall. 'Adult Cystic Fibrosis Centre, Belfast City Hospital, Belfast, UK; ${ }^{2}$ Northern Ireland Public Health Laboratory, Belfast City Hospital, Belfast, UK

10.1136/thoraxjnl-2016-209333.236

Introduction and objectives Mycobacterium abscessus pulmonary infection in patients with cystic fibrosis (CF) is associated with significant morbidity, and the prevalence is increasing. The cause of the apparent increase is unknown. Contributing factors may include the ageing CF population, and the potential for patientto-patient transmission. To date, there is a paucity of data describing the activity of common hospital biocides against this organism

Methods $M$. abscessus isolates $(n=13)$ were recovered from CF and non CF respiratory specimens. Seven commonly employed hospital biocides (Steri- ${ }^{\mathrm{TM}}$, Difficile-S ${ }^{\mathrm{TM}}$, Hydrex ${ }^{\mathrm{TM}}$, Cutan ${ }^{\mathrm{TM}}$, Stellisept ${ }^{\mathrm{TM}}$, Rely ${ }^{+} \mathrm{On}^{\mathrm{TM}}$ PeraSafe $^{\mathrm{TM}}$, Distaclor ${ }^{\mathrm{TM}}$ ) were assayed for their biocidal activity against M. abscessus. Fresh cultures of NTM were exposed to biocide in liquid medium as per manufacturers instruction and were immediately plated following the completion of the contact period. The mean concentration of NTM plated was $9.82 \times 10^{6}$ colony forming units $(\mathrm{CFU})$ (range: $\left.1.63 \times 10^{5}-1.12 \times 10^{8}\right)$. Additionally, the remaining bacteria/ biocide solution was enriched non-selectively in Mueller Hinton broth $\left(37^{\circ} \mathrm{C} / 1\right.$ week). Following this, growth of surviving bacteria was assessed with broth turbidity.

Results After appropriate exposure of NTM to biocide, all NTM isolates survived in Steri- $7^{\mathrm{TM}}$, Hydrex ${ }^{\mathrm{TM}}$, Stellisept ${ }^{\mathrm{TM}}$ and Rely ${ }^{+} \mathrm{On}^{\mathrm{TM}}$ PeraSafe ${ }^{\mathrm{TM}}$. One out of 13 NTM cultures was killed by Difficile-S ${ }^{\mathrm{TM}}$ and 1 by Distaclor ${ }^{\mathrm{TM}}$, representing a $5 \log$ kill. Two isolates were killed by Cutan ${ }^{\mathrm{TM}}$ again representing a $5 \log$ kill. Following enrichment, Stellisept ${ }^{\mathrm{TM}}$ showed the greatest biocidal activity with 11/13 isolates, whereas 2/13 cultures were killed by Distaclor ${ }^{\mathrm{TM}}$. All other biocide/culture combinations yielded growth.

Conclusions These data indicate that M. abscessus may persist after exposure to several commonly employed hospital biocides. Given the importance of effective infection prevention and control, further work is urgently needed to define unequivocal biocide contact treatments to ensure successful eradication. Acknowledgements SC is a CF Trust funded Clinical Fellow

\section{P94 \\ THE MANAGEMENT OF RESPIRATORY TRACT FUNGAL DISEASE IN CYSTIC FIBROSIS - A UK SURVEY OF CURRENT PRACTICE}

M Boyle, JE Moore, DG Downey. Northern Ireland Regional Adult Cystic Fibrosis Centre, Belfast, UK

10.1136/thoraxjnl-2016-209333.237

Aspergillus fumigatus is commonly found in the airways of patients with Cystic Fibrosis, (CF). Allergic Bronchopulmonary Aspergillosis, (ABPA), is the most recognised clinical condition associated with Aspergillus. The most widely used diagnostic criteria are from the Cystic Fibrosis Foundation Consensus Conference 2003. However, diagnosis remains challenging due to the overlap of classical symptoms and radiological features of ABPA and CF. There are a lack of clinical trials with clear outcomes to guide management of fungal disease, leading to variability between CF centres.

The aim of this survey was to assess the variability in current practice across the UK in diagnosis and management of fungal lung disease in CF patients.

A 21 question anonymous online survey was sent to 94 paediatric and adult CF consultants in the UK.

The response rate was $60.6 \%$ with 55 full and 2 partially completed surveys. Thirty-two respondents were adult physicians and twenty-five paediatricians. For a first diagnosis of ABPA 20 (35.1\%) treat with Prednisolone alone, 19 (33.3\%) use Prednisolone with Itraconazole capsules, 19 (33.3\%) use Prednisolone with Itraconazole liquid and 2 (3.5\%) choose Voriconazole.

Only $5(8.8 \%)$ treat with Prednisolone alone for a $1^{\text {st }}$ relapse, preferring Prednisolone with Itraconazole Liquid (33.3\%) or with Itraconazole capsules (24.6\%).

To reduce treatment, $21(36.8 \%)$ decrease steroids to zero over time and maintain azole therapy, 18 (31.6\%) stop the azole and steroid after a fixed time and 5 (8.8\%) stop azole after a fixed time and maintain a small steroid dose. Variations in specific therapies were reported, including the use of pulsed Methylprednisolone, Posaconazole, nebulised Amphotericin and Omalizumab.

Thirty-eight (66.7\%) respondents believe Aspergillus colonisation of the airway can cause clinical deterioration and 37 $(66.1 \%)$ would treat this. Scedosporium apiospermum infection has been diagnosed and treated by 35 (61.4\%) of respondents.

Results of this survey highlight significant differences in treatment regimes for ABPA, with increasing variation seen in the management of subsequent relapses. Respondent comments showed a wide range of opinions. This survey highlights the lack of evidence currently available to guide the management of $\mathrm{CF}$ fungal disease.

\section{P95 EXPLORING THE TIMING OF HYPERTONIC SALINE (HTS) AND AIRWAYS CLEARANCE TECHNIQUES (ACT) IN CYSTIC FIBROSIS (CF): A CROSS OVER STUDY}

${ }^{1} \mathrm{~K}$ O'Neill, ${ }^{2} \mathrm{~F}$ Moran, ${ }^{3}$ I Bradbury, ${ }^{4} \mathrm{DG}$ Downey, ${ }^{4} \mathrm{~J}$ Rendall, ${ }^{5} \mathrm{MM}$ Tunney, ${ }^{1} \mathrm{JS}$ Elborn, ${ }^{6} \mathrm{JM}$ Bradley. 'Queen's University Belfast, Centre for Experimental Medicine, UK; ${ }^{2}$ School of Health Sciences, UIster University, UK; ${ }^{3}$ Frontier Science (Scotland) Ltd, UK; ${ }^{4}$ Belfast Health and Social Care Trust, UK; ${ }^{5}$ Queen's University Belfast, School of Pharmacy, UK; ${ }^{6}$ Queen's University Belfast, Clinical Research Facility, UK 
Background Streamlining the timing of treatments in CF is important to optimise adherence whilst ensuring efficacy. The optimal timing of HTS and ACTs is unknown.

Objectives This study hypothesised that ACTs after HTS would be more effective than ACTs during HTS as measured by lung clearance index (LCI). FEV1, sputum weight and patient perceptions were also compared.

Methods Adults with CF providing written informed consent were randomised (between days 10-14 of intravenous antibiotic course during a pulmonary exacerbation) to a crossover trial of ACTs after HTS inhalation or ACTs during HTS inhalation on alternate days. ACT treatment consisted of 10 cycles of active cycle of breathing technique using an Acapella ${ }^{\circledR}$. The physiotherapist collecting the outcome measures was blinded. Patients completed a Multiple Breath Washout (MBW) test to obtain LCI and spirometry at baseline and 90 mins post treatment. Sputum collection during 90 mins, ease of clearance and satisfaction with treatment was also recorded. Wilcoxon test was used and $\mathrm{p}<0.05$ was considered significant.

Results Fourteen subjects were recruited and 13 completed the study (mean [SD] age 33 [12], FEV1\% predicted 51 [22], LCI (no. turnovers) 14 [4]). Comparing the 2 treatments (ACT after HTS vs ACT during HTS), the change from baseline to 90 mins post treatment in LCI $(\mathrm{p}=0.71)$ and $\mathrm{FEV} 1 \%$ predicted $(p=0.97)$ was not significant. There was also no difference in sputum weight expectorated ( $\mathrm{p}=0.17)$, patient perceived ease of clearance $(p=0.33)$ or satisfaction $(p=0.28)$. The time taken for ACT during HTS was significantly shorter $(p=0.001)$.

Conclusions In this small study, ACTs after HTS was no more effective than ACTs during HTS.

\section{P96 PHYSIOTHERAPY MANAGEMENT OF ADULT PATIENTS WITH CYSTIC FIBROSIS ON INTENSIVE CARE UNITS (ICU) - A SURVEY OF UK PHYSIOTHERAPISTS}

F Cathcart, H Parrott, A Jones, N Simmonds. Adult Cystic Fibrosis Centre, Royal Brompton Hopsital, London, UK

\subsection{6/thoraxjnl-2016-209333.239}

Introduction and objectives Currently no guidelines or data exist on the physiotherapy management of adults with CF on the ICU. The aim was to explore the views and experiences of the specialist physiotherapists (SPs) managing adults with CF admitted to an ICU.

Methods An online survey was designed which included questions (open and closed) on staff confidence, communication, learning needs, extracorporeal membrane oxygenation (ECMO) and end of life care. The survey was sent to CF, ICU and transplant SPs across the UK.

Results 42 SPs responded (74\% response rate), 52\% (n = 22) adult CF, 40\% ( $\mathrm{n}=17)$ ICU and 7\% $(\mathrm{n}=3)$ transplant specialists. $73 \%(\mathrm{n}=30)$ had been specialists in their area for $>5$ years. $27 \%(\mathrm{n}=11)$ reported no CF admissions to their ICU in the last year, only $24 \%(n=10)$ had $>3$ in the last year. Physiotherapy care was shared between the ICU and CF SPs teams in $43 \%$ $(\mathrm{n}=18)$ of respondents. 90\% $(\mathrm{n}=37)$ felt this joint working was essential to optimise patient care. On a confidence scale of 1 - $10(1=$ low, 10 = high) the median (IQR) confidence score of SPs to manage patients with CF on ICU was 7 (7-9).

43\% ( $n=17)$ had experienced pre transplant patients with $\mathrm{CF}$ being invasively ventilated and reported that the challenges included airway clearance, weaning, inhalation therapy and nursing staff education. Of the 17 respondents who worked in ECMO centres, $57 \%(n=12)$ had never had a patient with CF on ECMO and 90\% had no physiotherapy ECMO protocol. Reported challenges were mobilisation, chest clearance, inhalation therapy and palliative care.

Of respondents 50\% $(\mathrm{n}=21)$ had managed a patient with CF who died on ICU. 79\% $(n=33)$ of respondents wanted more education on managing patients with CF on ICU with comments around, joint training, national guidelines being developed and the importance of collaborative care.

Conclusions The number of adults with CF admitted to ICU remains low nationally however SPs need to maintain competence and confidence in managing these complex patients. National, expert consensus guideline development, including a physiotherapy ECMO protocol would assist in ensuring equitable quality care in this setting.

\section{P97 GASTRO-OESOPHAGEAL REFLUX IN CYSTIC FIBROSIS}

1,2RW Lord, ' 1 JS Pearson, ' $P J$ Barry, ${ }^{1} \mathrm{PJ}$ Whorwell, ${ }^{1} \mathrm{RB}$ Jones, ${ }^{3} \mathrm{P}$ McNamara, ${ }^{4} \mathrm{R}$ Beynon, ${ }^{1,2}$ JA Smith, ${ }^{1}$ AM Jones. ${ }^{1}$ University Hospital South Manchester, Manchester, UK; ${ }^{2}$ University of Manchester, Manchester, UK; ${ }^{3}$ Alder Hey Hospital, Liverpool, UK; ${ }^{4}$ University of Liverpool, Liverpool, UK

\subsection{6/thoraxjnl-2016-209333.240}

Background Initial small studies using combined $\mathrm{pH}$ and impedance ( $\mathrm{pH}-\mathrm{MII})$ have suggested increased gastro-oesophageal reflux (GOR) in adult cystic fibrosis (CF) patients. Reflux episodes frequently reach the proximal oesophagus and occur whilst supine, which may predispose to micro-aspiration.

Aims To investigate the relationship between GOR and CF lung disease.

Methods We conducted a prospective observational study in stable adult CF patients undergoing 24 hour pH-MII. Reflux symptoms (using validated RESQ-7 questionnaire) and spirometry were also recorded.

Results 10/12 subjects recruited completed pH-MII the study (mean age 28.5 years, mean FEV1 48.8\% predicted, 100\% male). An increased number of reflux episodes per 24 hours were noted in $80 \%$ of participants (median 104.1, IQR 78.6 -164.4, normal range $<75$ episodes), with increased 'high-risk' proximal reflux or supine reflux noted in $60 \%$ (Table 1). In this small preliminary patient sample there were no significant correlations between FEV1\% and total, proximal or supine reflux episodes, or RESQ7 heartburn scores and any reflux measure.

$7 / 10$ subjects completed the study on a PPI and had data available for acid reflux $(\mathrm{pH}<4)$ parameters. $4 / 7$ had an abnormal acid exposure despite standard dose PPI therapy. 5/7 had prolonged acid reflux events; the longest was 66.5 minutes (see Table 1).

Conclusions In this preliminary data set, CF patients appear to have a pattern of GOR that puts them at risk of reflux microaspiration and have significant acid reflux despite antacid therapy. Reflux symptoms and spirometry were not related to reflux parameters.

The clinical implications of these findings are that standard PPI dosing maybe insufficient in CF patients to control acid reflux. In addition silent reflux aspiration maybe occurring and as such could be a potential area for future therapies. 